\section{INJECTABLE CHITOSAN HYDROGEL FOR LOCALIZED DELIVERY OF IMMUNE CHECKPOINT INHIBITORS}

Siena Mantooth*, David Zaharoff, Siena Mantooth. North Carolina State University, Cary, $N C$, USA

Background Systemic delivery of checkpoint inhibitors risks the development of immune-related adverse events (irAEs) in up to $85 \%$ of patients. ${ }^{1}$ Localized delivery methods with slow-release kinetics have the potential to avoid systemic exposure and reduce irAEs. Direct tumor injection is extremely difficult, as saline-based solutions are rapidly excluded from the high-pressure tumor environment. Utilizing hydrogels as a delivery medium and local depot can address this shortcoming. To this end, we developed an injectable chitosan-based hydrogel for intratumoral delivery of checkpoint antibodies.

Methods

Hydrogel Low-viscosity, 80\% deacetylated chitosan (Heppe Medical Chitosan; Halle, Germany) was reacted with 1-ethyl3-(-3-dimethylaminopropyl) carbodiimide hydrochloride (EDC) and N-hydroxysuccinimide (NHS) in the presence of $\beta$-glycerophosphate at room temperature for 48 hours. The mixture was then washed with ethanol and dried at $60^{\circ} \mathrm{C}$. The resulting solid was dissolved in phosphate buffered saline (PBS) at concentrations from 35-70 mg/mL.In vitro release. $300 \mu \mathrm{g} / \mathrm{mL}$ bovine serum albumin (BSA) labeled with fluorescein-5-isothiocyanate (FITC) as a model protein drug was loaded into the hydrogel. The hydrogel was injected through a $28 \mathrm{~g}$ needle and incubated with PBS. Samples were taken over a week period. Release kinetics were analyzed by fitting fluorescence data to zero-order, first-order, and Korsmeyer-Peppas models. To visualize retention after injection, dye-loaded hydrogels or dye in PBS alone were injected into a $0.6 \mathrm{wt} \%$ agar tissue phantom.In vivo imaging and tumor treatment. Flank MC38 tumors will be established in $\mathrm{C} 57 \mathrm{BL} / 6$ mice. At tumor volumes of $50-100 \mathrm{~mm} 3,200$ ug of fluorescently labeled aCTLA-4 and aPD-L1 included in the chitosan hydrogel will be delivered intratumorally. Images will be captured using an In Vivo Imaging System (IVIS). Antitumor activity will be assessed in a separate cohort using unlabeled antibodies.

Results The chitosan hydrogel was found to be injectable in needles as thin as $28 \mathrm{~g}$. After exiting the needle, the hydrogel reformed (figure 1A). Upon injection into the tissue phantom, dyed PBS immediately leaked out, primarily through the needle track, while the dyed hydrogel was retained (figure 1B). In vitro release studies demonstrated long-term, nearly zeroorder, week-long sustained release (figure 1C). In vivo retention and tumor treatment studies are ongoing.

Conclusions A novel injectable chitosan hydrogel was found to provide sustained release of a large model protein over a 1-2 week period with favorable in vitro kinetics. Importantly, this hydrogel can be engineered to provide faster or slower release as needed. Ongoing studies in vivo will quantify intratumoral retention, systemic dissemination, and antitumor activity.

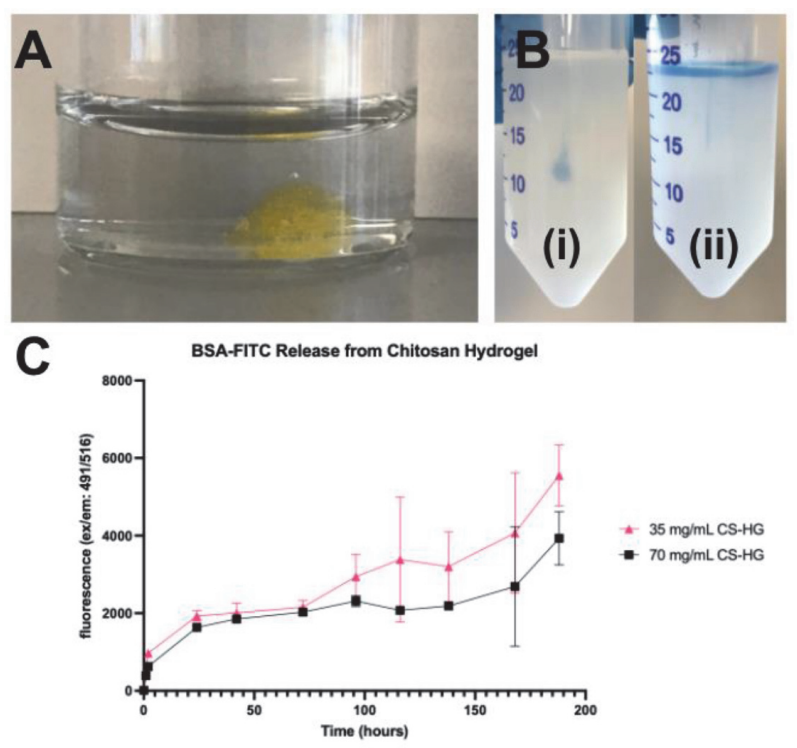

Abstract 259 Figure 1 Injectable chitosan hydrogel. (A) Re-formed BSA-FITC hydrogel in 1x PBS; (B) (i) Retained hydrogel in agar tissue phantom, (ii) Excluded 1x PBS in agar tissue phantom; (C) Release kinetics in $1 \mathrm{x}$ PBS.

Acknowledgements This work is supported by the National Science Foundation Graduate Research Fellowship.

\section{REFERENCE}

1.. Hommes J, Verheijden R, Suijkerbuijk K, Hamann D. Biomarkers of checkpoint inhibitor induced immune-related adverse events - a comprehensive review. Front Oncol 2021;10:1-16.

Ethics Approval Animal use was in compliance with the Public Health Service Policy on Human Care and Use of Laboratory Animals. All experiments involving laboratory animals were approved by the Institutional Animal Care and Use Committee at North Carolina State University (Protocol \#19-795).

http://dx.doi.org/10.1136/jitc-2021-SITC2021.259 